\section{Transrektale Prostatabiopsien: Hat Ciprofloxacin zur Infektprophylaxe bald ausgedient?}

\author{
Bei transrektalen Prostatabiopsien wird standardmäßig eine antibiotische \\ Prophylaxe mit Ciprofloxacin durchgeführt. Doch offenbar gibt es wirksamere \\ Alternativen.
}

$\mathrm{M}_{\mathrm{s}}^{\mathrm{i}}$ it der Zunahme Fluorchinolon-resistenter Keime in den letzten Jahren stieg die Rate infektbedingter Komplikationen nach transrektaler Prostatabiopsie. Die schlechte Resistenzlage reißt eine große Lücke, da sich von den heute verfügbaren Antibiotika nur wenige zur prophylaktischen Anwendung eignen und neue Wirkstoffe in absehbarer Zeit nicht verfügbar sind. Ob mit FosfomycinTrometamol (FT) diese Lücke zu schließen ist, hat ein Team um den italienischen Urologen Tommaso Cai überprüft und beide Wirkstoffe hinsichtlich Wirksamkeit und Verträglichkeit verglichen.

Cai et al. werteten retrospektiv die Daten von insgesamt 1.109 Männern aus, die sich zwischen März und September
2015 in einem von sieben großen urologischen Zentren in Italien einer transrektalen Prostatabiopsie unterzogen hatten. 632 Männer hatten zur Infektprophylaxe FT eingenommen, 477 Ciprofloxacin (CIP). Infektbedingte Komplikationen wurden bei insgesamt 83 Männern vermerkt: 72 entwickelten einen symptomatischen Harnwegsinfekt, elf eine Urosepsis. Erwähnenswert ist, dass von den 72 Harnwegsinfekten 53 auf das Konto Fluorchinolon-resistener Erreger gingen.

Tatsächlich stellte sich FT als gute und sogar wirksamere Alternative heraus: 12,9 \% der Männer, die Ciprofloxacin eingenommen hatten, entwickelten im Anschluss an die transrektale Prostatabiopsie einen symptomatischen Harnwegsin- fekt, aber nur 1,6\% der FT-Anwender. Auch im Hinblick auf das Sepsisrisiko schnitt FT besser ab $(\mathrm{p}<0,001)$. Während in der CIP-Gruppe 1,8 \% der Patienten wegen einer Sepsis behandelt werden mussten, waren es in der FT-Gruppe nur 0,3 \%. Das Epoxid-Antibiotikum verursachte zudem etwas weniger Nebenwirkungen, der Unterschied war jedoch statistisch nicht signifikant $(0,6 \%$ vs. $0,4 \%$; $\mathrm{p}=0,94)$.

Fazit: Für die italienischen Urologen steht somit fest: FT eignet sich durchaus zur perioperativen Infektprophylaxe bei transrektaler Prostatabiopsie und ist eine gute Alternative zu Ciprofloxacin. Als entscheidenden Vorteil werten die Studienautoren die bislang noch geringen Resistenzraten sowie die fehlenden Kreuzund Parallelresistenzen in Bezug auf andere häufig verwendete Antibiotika.

Dr. Dagmar Kraus

Cai T et al. Antimicrobial prophylaxis for transrectal ultrasound guided prostate biopsy: fosfomycin trometamol, an attractive alternative. World J Urol. 2017; 35: 221-8.

\section{Ureterstents sollten nicht länger als einen Monat liegen}

\author{
Wird Patienten vor einer Ureteroskopie zur Steinentfernung ein Ureterstent \\ geschoben, erhöht sich die Gefahr für eine postoperative Sepsis - und zwar \\ umso mehr, je länger der Stent liegt.
}

n Studien erreicht der Anteil der Patienten mit Ureterstents vor einer Ureteroskopie zur Steinenfernung bis zu $68 \%$. Gründe dafür können die Notwendigkeit einer notfallmäßigen Drainage bei Obstruktion oder Infektion oder auch die Schmerzentlastung von Patienten sein, bei denen die primäre Therapie nicht möglich ist, etwa aufgrund von Begleitkrankheiten. Nebenwirkungsfrei ist die Stenteinlage nicht. Neben den erwähnten Infektionsrisiken sind Flankenschmerzen, Stauungssymptome, Hämaturie und Dysurie häufige Beschwerden.

Das Einlegen von Ureterschienen ist mit einigen Vorteilen für das endoskopische Management einer Urolithiasis verbunden. Beispielsweise erhöht sich der Anteil der Patienten, die steinfrei bleiben. Auch treten während des Eingriffs seltener Komplikationen auf. Dennoch ist ein Ureterstent ein Fremdkörper in den Harnwegen, der das Risiko für eine bakterielle Kolonisation erhöht. Nach ein paar Wochen sind die meisten Stents mit Keimen besiedelt. Welche Konsequenzen das für das Risiko der Patienten hat, an postoperativer Urosepsis zu erkranken, haben israelische Urologen um Amihay Nevo untersucht.

1.256 Patienten im medianen Alter von 56 Jahren, die sich einer ureteroskopischen Steinextraktion unterziehen mussten, waren an der Untersuchung beteiligt. 601 von ihnen hatten vor dem
Eingriff einen Ureterstent gelegt bekommen. Die Rate an postoperativer Sepsis betrug 1,2 \% für Patienten ohne und $4,7 \%$ für Patienten mit Stent. Allerdings änderten sich die Anteile von Stentpatienten mit Sepsis je nach Verweildauer der Harnleiterschienung. Für eine Liegedauer von ein, zwei, drei und mehr als drei Monaten waren Raten von 1,1\%, $4,9 \%, 5,5 \%$ und $9,2 \%$ zu verzeichnen.

Fazit: Eine Liegedauer von Harnleiterschienen, ab der es mit dem Sepsisrisiko steil bergauf geht, konnten Nevo et al. nicht ausmachen. Sie empfehlen jedoch, Ureterstents höchstens 30 Tage liegen zu lassen. Bei einer kürzeren Liegedauer beträgt die Sepsisrate 1,1 \%. Werden hingegen 30 Tage überschritten, erhöht sich der Anteil von Patienten, die nach der Ureteroskopie eine Sepsis entwickeln, alle Liegedauern von mehr als einem Monat zusammengenommen - auf 6,2\%.

Dr. Robert Bublak

Nevo A, et al. Ureteral stent dwelling time: a risk factor for post-ureteroscopy sepsis. BJU Int. 2017; doi: 10.1111/bju.13796. 